\title{
White matter characteristics of motor, sensory and interhemispheric tracts underlying impaired upper limb function in children with unilateral cerebral palsy
}

Lisa Mailleux ${ }^{1}$, Cristina Simon-Martinez ${ }^{1}$, Ahmed Radwan ${ }^{2}$, Jeroen Blommaert ${ }^{3}$, Jolien Gooijers ${ }^{4}$, Nicole Wenderoth $^{4,5}$, Katrijn Klingels ${ }^{1,6}$, Els Ortibus ${ }^{7}$, Stefan Sunaert ${ }^{2}$, Hilde Feys ${ }^{1}$

${ }^{1} \mathrm{KU}$ Leuven, Department of Rehabilitation Sciences, Leuven, Belgium

${ }^{2} \mathrm{KU}$ Leuven, Department of Imaging and Pathology, Leuven, Belgium

${ }^{3}$ KU Leuven, Department of Oncology, Leuven, Belgium

${ }^{4}$ KU Leuven, Department of Movement Sciences, Leuven, Belgium

${ }^{5}$ Neural Control of Movement Lab, Department of Health Sciences and Technology, ETH Zurich, Zurich, Switzerland

${ }^{6}$ UHasselt, BIOMED, Rehabilitation Research Center (REVAL), Diepenbeek, Belgium

${ }^{7} \mathrm{KU}$ Leuven, Department of Development and Regeneration, Leuven, Belgium

Corresponding author: Lisa Mailleux

$\underline{\text { lisa.mailleux@kuleuven.be }}$

+3216379385

\section{Acknowledgements}

We acknowledge all parents and their children for their participation in this study. We also thank Jasmine Hoskens for her assistance during the assessments. Lastly, we would like to acknowledge, dr. Annouschka Laenen and dr. Anna Ivanova, from the Leuven Biostatistics and Statistical Bioinformatics Centre (LBioStat), KU Leuven for their advice regarding the statistical analyses.

\footnotetext{
“"This is a pre-print of an article published in Brain Structure and Function. The final authenticated version is available online at: https://doi.org/10.1007/s00429-020-02070-1."
} 


\begin{abstract}
This study explored the role of lesion timing (periventricular white matter versus cortical and deep grey matter lesions) and type of corticospinal tract (CST) wiring pattern (contralateral, bilateral, ipsilateral) on white matter characteristics of the CST, medial lemniscus, superior thalamic radiation and sensorimotor transcallosal fibers in children with unilateral cerebral palsy (CP), and to determine the association with upper limb function. Thirty-four children (mean age $10 \mathrm{y} 7 \mathrm{~m} \pm 2 \mathrm{y} 3 \mathrm{~m}$ ) with unilateral CP underwent a comprehensive upper limb evaluation and diffusion weighted imaging (75 directions, b-value 2800). Streamline count, fractional anisotropy and mean diffusivity were extracted from the targeted tracts and asymmetry indices were additionally calculated. Transcranial magnetic stimulation was applied to assess the CST wiring pattern. Results showed a more damaged CST in children with cortical and deep grey matter lesions $(N=10)$ and ipsilateral CST projections $(N=11)$ compared to children with periventricular white matter lesions $(N=24 ; \mathrm{p}<0.02)$ and contralateral CST projections $(N=9 ; \mathrm{p}<0.025)$, respectively. Moderate to high correlations were found between diffusion metrics of the targeted tracts and upper limb function $(r=0.45-0.72 ; p<0.01)$. Asymmetry indices of the CST and sensory tracts could best explain bimanual performance $(74 \%, p<0.0001)$ and unimanual capacity $(50 \%, p=0.004)$. Adding lesion timing and CST wiring pattern did not further improve the model of bimanual performance, while for unimanual capacity lesion timing was additionally retained $(58 \%, p=0.0002)$. These results contribute to a better understanding of the underlying neuropathology of upper limb function in children with unilateral CP and point towards a clinical potential of tractography.
\end{abstract}

Keywords: unilateral cerebral palsy; upper extremity; brain injuries; diffusion magnetic resonance imaging; transcranial magnetic stimulation. 


\section{Introduction}

The developing human brain possesses intriguing compensatory mechanisms to retain sensorimotor functions after a lesion occurring early in brain development (Staudt 2010). Hence, neuroimaging studies in children with early brain lesions, such as those with cerebral palsy (CP), have increased exponentially in an attempt to unravel how the underlying neuropathology determines their level of functioning. CP is a nonprogressive brain disorder, causing a range of symptoms that vary greatly amongst these children (Colver, Fairhurst, and Pharoah 2014). It has been proposed that their clinical variability is at least partially caused by differences in characteristics of the underlying brain lesion. In children with unilateral CP, predominant unilateral brain lesions will elicit sensorimotor symptoms at one side of the body. The largest part of these children have average cognitive abilities and achieve independent walking (Beckung et al. 2008; Reid et al. 2018). However, the limitations of their upper limb are more variable and may still adversely affect their independence in life (Klingels et al. 2012). Moreover, upper limb function is not solely determined by structural and functional limitations, but also by practice, preference and strategy (Hoare et al. 2018). Hence, understanding the clinical variability of the upper limb and its best treatment options within this population is a major clinical concern.

Studies investigating structure-funtion relationships often classify children with CP based on specific brain lesion characteristics. Most commonly, children with CP are classified based on the timing of the lesion: i.e. cortical maldevelopments (occurring in the first and second trimester of gestation), periventricular white matter (PWM) lesions (early third trimester) or cortical and deep grey matter (CDGM) lesions (around term age) (Krägeloh-Mann and Horber 2007). Secondly, in children with unilateral CP also the corticospinal tract (CST) wiring pattern may vary. During normal brain development, the CST sprouts bilaterally from each hemisphere to both hands. From the third trimester of pregnancy onwards, the ipsilateral tracts gradually disappear (Eyre et al. 2007). However, in case of an early unilateral brain lesion, ipsilateral tracts can be partially or completely preserved, resulting in a bilateral or ipsilateral CST wiring pattern innervating the impaired hand (Staudt 2010; Eyre et al. 2007). Both variables have been shown to relate with upper limb function in children with unilateral CP (Staudt 2010), though a large part of the variability in upper limb function still remains unexplained (Feys et al. 2010; Staudt et al. 2004). Hence, diffusion weighted imaging is increasingly used in children with CP to obtain more precise information on deficits in brain white matter (Son et al. 2007). Recent studies already demonstrated the negative relation between damaged white matter pathways of the motor, sensory and interhemispheric tracts (i.e. corpus callosum) and upper limb function in children with unilateral CP (Mailleux et al. 2020). Still, correlations were mostly low to moderate, suggesting that these factors are also not sufficient as a predictor for motor outcome. Moreover, the majority of existing studies focused on one particular tract.

This raises the question whether combining multiple structural brain lesion characteristics, retrieved from different imaging techniques would further enhance our understanding of the neuropathology underlying upper limb dysfunction in children with unilateral CP. Furthermore, it remains unknown whether white 
matter characteristics differ depending on the aforementioned classification groups (i.e. lesion timing and CST wiring patterns). Gaining insights in these associations would contribute to a better understanding of the relationship between specific brain lesion characteristics and upper limb function in children with unilateral CP. Hence, the first aim of this study was to compare white matter characteristics of the CST, medial lemniscus, superior thalamic radiations and the sensorimotor transcallosal fibers between children with different lesion timings and CST wiring patterns. Secondly, we systematically assessed the relationship between the diffusion metrics of these tracts and upper limb motor and sensory function. Lastly, we investigated which set of diffusion metrics explained most of the variance in bimanual performance, and investigated whether adding lesion timing and type of CST wiring pattern would improve the regression model. We hypothesized that damage to white matter tracts would be more pronounced in children with CDGM lesions and an ipsilateral or bilateral CST wiring pattern compared to children with PWM lesions and a contralateral CST wiring pattern, respectively. We further hypothesized that diffusion metrics of the CST would be the most important determinant for bimanual performance and that sensory and/or interhemispheric connectivity would play an additional role. Finally, it was expected that adding lesion timing and type of CST wiring pattern would further increase the amount of explained variance.

\section{Materials and methods}

\section{Participants}

Forty-seven children with spastic unilateral CP (aged 5-15 years) were recruited for this study between May 2014 and April 2017 via the CP-care program of the University Hospitals Leuven (Belgium). Exclusion criteria were: (1) botulinum toxin-A injections in the six months prior to testing, (2) upper limb surgery and (3) any contraindications for MRI. Eight children were excluded due to excessive head motion and four due to the inability of generating the CST, medial lemniscus and superior thalamic radiation in the lesioned hemisphere nor the sensorimotor transcallosal fibers. One child was classified as having a cortical maldevelopment, and was excluded for further analyses. Of the remaining 34 children, 24 were classified in the PWM group and 10 in the CDGM group. Regarding type of CST wiring pattern, 9 children had contralateral CST projections (8 PWM and 1 CDGM), 10 bilateral CST projections (5 PWM, 5 CDGM) and 11 ipsilateral CST projections (8 PWM, 3 CDGM). Due to the presence of epilepsy $(N=2)$ or due to refusal $(N=2)$, we were unable to perform the TMS assessment in four children. Average age at time of the assessments was 10 years and 3 months (SD 2 years and 7 months). A detailed overview of patient's characteristics is presented in Supplementary Material 1. The protocol was approved by the Ethical Committee of the University Hospitals Leuven (S55555, S56513) and parental written informed consent was obtained for all children prior to participation, according to the Declaration of Helsinki. Children aged 12 years or above were additionally asked for their assent prior to participation. 


\section{Procedure}

All children underwent a comprehensive upper limb evaluation, including a clinical assessment of motor and sensory impairments, as well as an evaluation of bimanual performance and unimanual capacity at the Clinical Motion Analysis Laboratory of the University Hospitals Leuven. Children were assessed by three well-trained physiotherapists who were routinely involved in the clinical evaluation of children with CP. At the same day or with a time interval of maximum four months, all children underwent a scanning protocol including structural and diffusion weighted MRI scans, as well as transcranial magnetic stimulation (TMS) to determine the CST wiring pattern.

\section{Clinical assessment}

Sensorimotor impairments were evaluated according to a standardized and reliable protocol (Klingels et al. 2010), including muscle tone, muscle strength, grip force and sensory function. Muscle tone was assessed in six muscle groups at the level of the shoulder (adductors, internal rotators), elbow (flexors, pronators), wrist and hand (wrist and finger flexors) (total score; 0-24) using the Modified Ashworth Scale (Bohannon and Smith 1987). Muscle strength was measured in four muscle groups at the level of the shoulder (abductors), elbow (extensors and supinators) and wrist (extensors) (total score; 0-20) using the Medical Research Council rating. Grip force was evaluated with the Jamar dynamometer (Lafayette Instrument Company, Lafayette, IN). The ratio of the mean of three maximum contractions of the impaired versus the less impaired hand was used for further analyses. Sensory assessments included two-point discrimination (TPD) and stereognosis. TPD was evaluated at the distal phalanx of the index finger as the minimal distance at which one or two points were correctly distinguished using an aesthesiometer®. Stereognosis was assessed through tactile identification of six out of twelve familiar objects; three of six objects matched in pairs (pencil/ pen, coin/button, paperclip/safety pin) and three of six differing objects (key, clothespin, marble, comb, spoon, ball).

To evaluate bimanual performance, the Assisting Hand Assessment (AHA) (Krumlinde-Sundholm et al. 2007; Holmefur and Krumlinde-Sundholm 2016) was used. The AHA assesses the spontaneous use of the impaired hand in bimanual activities during a semi-structured play session which is video-recorded. Afterwards, 22 items describing object-related hand actions were scored and converted to 0-100 logit-based AHA units (Holmefur and Krumlinde-Sundholm 2016). The Melbourne Assessment 2: a test of unilateral upper limb function (MA2) and the Jebsen-Taylor Hand Function Test (JTHFT) were used to assess unimanual capacity of the impaired hand. The MA2 is a criterion-referenced test that evaluates four elements of upper limb movement quality: range of motion, accuracy, dexterity and fluency (Randall et al. 2014). It contains 14 unimanual tasks which are video-recorded for subsequent scoring. Raw scores were converted to a percentage score for each of the four sub-scales. The JTHFT evaluates movement speed during the execution of six unimanual tasks (Taylor, Sand, and Jebsen 1973). The ratio of the impaired versus the less impaired hand was used for further analyses. Finally, manual ability during daily life activities was assessed 
with the ABILHAND-kids questionnaire filled in by the parents (Arnould et al. 2004). Raw scores were converted to logits. All clinical scales are found to be reliable and valid (Krumlinde-Sundholm et al. 2007; Holmefur and Krumlinde-Sundholm 2016; Randall et al. 2014; Taylor, Sand, and Jebsen 1973; Arnould et al. 2004).

\section{Medical imaging}

A 3.0 Tesla MRI scanner (Achieva, Philips Medical Systems, Best, The Netherlands) with a 32-channel head coil was used for image acquisition, using a child friendly approach including a familiarization protocol with the children up to ten years of age (Verly et al. 2018) and a presentation of a video during scanning. The imaging protocol consisted of a T1-weighted anatomical image acquired using three-dimensional T1weighted turbo field echo with the following parameters: slice gap 0, TE/TR/TI 4.6/9.7/866 ms, FOV 250FHx250RLx192AP mm³ $0.98 \times 0.98 \times 1.2 \mathrm{~mm}^{3}$ voxel size. Diffusion weighted images were acquired using the following parameters: single-shot spin-echo Echo Planar Imaging; slice thickness $2.5 \mathrm{~mm}$, TE/TR 90/7800 ms, anterior-posterior phase encoding direction with an inplane parallel acquisition factor (i.e. SENSE factor) of 2.5 (Pruessmann et al. 1999), number of sagittal slices 58 , voxel size $2.5 \times 2.5 \times 2.5 \mathrm{~mm}^{3}$, 75 uniformly distributed diffusion directions with a b-value of $2800 \mathrm{~s} / \mathrm{mm}^{2}$. In addition, 10 non-diffusion weighted images were obtained.

\section{MRI processing}

Pre-processing and tractography were performed using FSL (version 5.0.1) and ExploreDTI (version 4.8.6) (Leemans et al. 2009). The T1 images were first reoriented to standard orientation using fslreorient 2 std and skull stripped using the optiBET script (Lutkenhoff et al. 2014), which optimizes brain extraction for T1 images with large brain pathologies using the FSL BET tool. The reoriented skull stripped images were then rigidly linearly aligned (6 degrees of freedom) to MNI space using FSL FLIRT. Diffusion weighted images were visually inspected for artefacts from different orthogonal views. ExploreDTI was used for calculation of the initial tensor images, which were then corrected for Gibbs ringing (Perrone et al. 2015), eddy current induced distortions, subject motion, and EPI induced geometrical distortions. Echo planar imaging distortions were corrected by elastic registration to the preprocessed $\mathrm{T} 1$ images. In addition, outliers were detected and removed using REKINDLE (Tax et al. 2015). Diffusion image correction also included a bmatrix rotation (Leemans et al. 2007). Participants with more motion than $2.5 \mathrm{~mm}$ in one single frame (original voxel size; translational parameter) were excluded. Secondly, whole brain deterministic tractography was performed in ExploreDTI with a seed point resolution of $1.5 \times 1.5 \times 1.5 \mathrm{~mm}$, fractional anisotropy threshold of 0.2, a maximum angle of 35 degrees, fiber length between 10-500 $\mathrm{mm}$ and using random perturbation of seed points. The whole brain tractography data was subsequently dissected using manually drawn regions of interest (ROIs) for the delineation of each fiber bundle in each participant, including the CST, medial lemniscus, superior thalamic radiations and the sensorimotor transcallosal 
fibers. At least two ROIs were manually drawn to identify streamlines for each fiber bundle according to specific anatomical landmarks (see Figure 1) (Hofer and Frahm 2006; Mori et al. 2008; Thomas et al. 2005).

\section{Data analysis}

For the CST, the initial 'AND' ROI was placed in the upper pons with 'AND' ROIs around the ipsilateral PLIC and Rolandic area in the axial plane (Mori et al. 2008). For the medial lemniscus, the initial 'AND' ROI was drawn on the posterior column at the level of the pons, with 'AND' ROIs around the ipsilateral cerebral peduncle and Rolandic area in the axial plane (Mori et al. 2008). For the superior thalamic radiations, the initial 'AND' ROI was placed around the thalamus with an 'AND' ROI around the ipsilateral parietal cortex in the axial plane (Thomas et al. 2005). For the sensorimotor transcallosal fibers, an initial 'AND' ROI was drawn around the posterior half of the sensorimotor transcallosal fibers in the sagittal plane, with 'AND' ROIs around the Rolandic area of each hemisphere in the axial plane (Hofer and Frahm 2006). Spurious streamlines, not belonging to the anticipatory trajectories were trimmed using exclusion ROI's. Finally, streamline count, fractional anisotropy and mean diffusivity averaged along each tract were calculated. In addition, asymmetry indices of these values were calculated as: $A I=(D-L) /(D+L)$, where ' $D$ ' represents the diffusion metric of the dominant hemisphere (i.e. less affected hemisphere) and ' $\mathrm{L}$ ' of the lesioned hemisphere. Values of these asymmetry indices can vary between ' -1 ' to ' 1 '. A value closer to ' 0 ' equals more symmetry between both hemispheres. For streamline count, only the asymmetry index was used as streamline counts are not comparable between subjects (Jones, Knösche, and Turner 2013). Moreover, streamline count does not reflect the actual amount of fibers and should thus be interpreted as a measure of tract quality (Jones, Knösche, and Turner 2013). The degree of overlap between all CST's, medial lemniscus and superior thalamic radiation of the same hemisphere was quantified using dice coefficients (Dice 1945), calculated with in-house written Matlab scripts. These calculations revealed low dice coefficients , indicating low similarity between homologous fiber bundles of the CST and superior thalamic radiation $(0.01 \pm 0.03$ to $0.02 \pm 0.03)$ and medial lemniscus and superior thalamic radiation $(0.08 \pm 0.08$ to $0.11 \pm 0.09)$. Dice coefficients between the CST's and medial lemniscus showed a slightly higher overlap ( $0.23 \pm 0.14$ to $0.25 \pm 0.13$ ), which is not surprising as these sensorimotor fibers overlap in the brain and are mainly delineated from each other in the brainstem (Mai and Paxinos 2011).

[Insert Figure 1 here]

Fig. 1. Manual delineation of the regions of interest in yellow and tracking results for $(A)$ the sensorimotor transcallosal fibers, (B) the corticospinal tract, (C) medial lemniscus and (D) superior thalamic radiations.

These a priori determined instructions, defined by two neuroradiologists (AR, SS), were followed and consistently applied for each participant. Intraclass correlation coefficients (ICC) were first calculated on 15 
randomly selected participants to evaluate interrater reliability of two independent raters (LM, AR) using streamline count, fractional anisotropy and mean diffusivity of all tracts. ICC's for interrater reliability were high for the CST (0.88 to 0.998 ), the medial lemniscus (0.81 to 0.998 ), the superior thalamic radiations ( 0.79 to 0.97 ) as well as the sensorimotor transcallosal fibers ( 0.85 to 0.98$)$. Mean ICC's $(95 \% \mathrm{CI})$ for each DTI metric for each tract are provided in Supplementary Material 2. In addition, the degree of overlap between both raters was quantified using dice coefficients, revealing good coefficients for the CST (less affected CSTs: $0.84 \pm 0.08$; lesioned CSTs: $0.79 \pm 0.26$ ), medial lemniscus (less affected: $0.83 \pm 0.1$; lesioned: $0.74 \pm 0.19)$ and sensorimotor transcallosal fibers (0.77 \pm 0.15$)$ (Zijdenbos et al. 1994). Dice coefficients of the superior thalamic radiation in the less affected hemisphere were also good $(0.73 \pm 0.18)$. In the lesioned hemisphere, dice coefficients of this tract were rather moderate $(0.62 \pm 0.21)$. Subsequently, one rater (LM) analyzed all other participants.

\section{Transcranial Magnetic Stimulation}

Participants without contraindications (e.g. ventriculo-peritoneal shunt, epilepsy) underwent the TMS assessment. TMS was performed by an experienced assessor (CSM) to identify the CST wiring pattern innervating the impaired hand: i.e. contralateral, bilateral or ipsilateral. TMS was conducted with a Magstim 200 stimulator (Magstim Ltd, Whitland, Wales, UK) connected to a 70mm figure-eight coil. Motor evoked potentials from the adductor pollicis brevis muscles were recorded from both hands using a Bagnoli electromyography system with a single differential surface electrode (Delsys Inc, Natick, MA, USA) attached to each muscle. A Micro1401-3 acquisition unit and Spike software version 4.11 (Cambridge Electronic Design Limited, Cambridge, UK) were used to synchronize the TMS stimuli and the electromyography data acquisition.

The protocol defined by Staudt et al. (2002) was followed. TMS was applied over the cortical representation of the hand in order to identify the hotspot (i.e. optimal point to stimulate) and resting motor threshold for the abductor pollicis brevis muscles. Stimulation was started at 30\% and gradually increased in steps of 5\%. The resting motor threshold was defined as the minimum intensity needed to obtain 5 out of 10 motor evoked potentials of at least $50 \mu \mathrm{V}$ in the correspondent muscle. First, the less affected hemisphere was stimulated to identify contralateral CST projections to the less impaired hand. Secondly, stimulation of the less affected hemisphere was increased up to $100 \%$ of the maximum stimulator output to search for ipsilateral CST projections to the impaired hand. Third, the lesioned hemisphere was stimulated to search for contralateral CST projections to the impaired hand. The absence of ipsilateral responses when stimulating the less affected hemisphere indicated that the impaired upper limb was mainly controlled by the lesioned hemisphere, i.e. 'contralaterally wired'. If motor evoked potentials were present during stimulation of both hemispheres, the child was classified as 'bilateral wired'. In case motor evoked potentials at the impaired hand could only be identified when the less affected hemisphere was stimulated, the child was categorized as 'ipsilateral wired'. 


\section{Statistical analysis}

The Shapiro-Wilk test was used to assess data distribution. To investigate differences in diffusion metrics (fractional anisotropy and mean diffusivity) of the CST, medial lemniscus, superior thalamic radiations of both hemispheres and the sensorimotor transcallosal fibers depending on lesion timing (i.e. PWM or CDGM lesions) or CST wiring pattern (i.e. contralateral, bilateral or ipsilateral), we used an ANCOVA with age as covariate. Comparisons of the asymmetry indices between lesion timing were done with an unpaired t-test in case of normally distributed data (i.e. asymmetry index mean diffusivity CST and medial lemniscus) or with a Mann-Whitney $U$ test in case of not normally distributed data. To compare the asymmetry indices between type of CST wiring pattern, an ANOVA was used in case of normally distributed data (asymmetry index fractional anisotropy CST; asymmetry index mean diffusivity CST and medial lemniscus) and for not normally distributed data with a Kruskall-Wallis test . Post-hoc significance level was adjusted according to Bonferroni for comparisons between the three CST wiring patterns.

The relation between upper limb function measures and diffusion metrics (fractional anisotropy and mean diffusivity) of the tracts of only the lesioned hemisphere and the sensorimotor transcallosal fibers was investigated using partial correlations ( $r$ ) with age as covariate. Depending on the type of data, spearman's rank $\left(r_{s}\right)$, biserial $\left(r_{b}\right)$ or pearson's $\left(r_{p}\right)$ correlation coefficients were calculated between the upper limb function measures and the asymmetry indices. Spearman's rank $\left(r_{s}\right)$ correlations coefficients were calculated between two not-normally distributed variables, biserial $\left(\mathrm{r}_{\mathrm{b}}\right)$ correlation coefficients between a normally and not-normally distributed variable and pearson's $\left(r_{p}\right)$ correlation coefficients between two normally distributed variables. The level of significance was set at 0.01 based on the Bonferroni correction, due to the high amount of correlations. We hypothesized that at least one of five correlations between the five DTI metrics (fractional anisotropy, mean diffusivity and the three asymmetry indices) of one specific tract and each clinical measure is different from zero, resulting in a corrected $\mathrm{p}$-value of $0.01(\mathrm{p}=0.05 / 5)$. Moreover, correlations will be mainly interpreted according to their strength as this is clinically more meaningful (Schober, Boer, and Schwarte 2018). Absolute correlation coefficients $<0.20$ were considered as a negligible correlation, $0.21-0.39$ as low, $0.40-0.69$ as moderate, $0.70-0.89$ as high, and $>0.90$ as very high correlation (Ariola 2006).

A multiple regression analysis using the R-Square Selection Method (Freund and Littell 2000) was performed to identify the best set of diffusion metrics explaining the largest variance in bimanual performance (i.e. AHA) as well as in unimanual capacity (i.e. Jebsen Taylor Hand Function Test of the impaired hand). Due to the relatively small sample size, we selected maximally three independent variables to explain bimanual performance in order to avoid overfitting of the model (i.e. one independent variable for ten participants) (Harrell, Lee, and Mark 2005). Two models were tested, one model with fractional anisotropy and mean diffusivity of the lesioned CST, medial lemniscus, superior thalamic radatiations and the sensorimotor transcallosal fibers, and a second model with the asymmetry indices of fractional 
anisotropy, mean diffusivity and streamline count of the CST, medial lemniscus and superior thalamic radiations along with fractional anisotropy and mean diffusivity of the sensorimotor transcallosal fibers. Secondly, we explored whether lesion timing and/or CST wiring pattern would have an additional value in explaining the variance in bimanual performance, resulting in a third and fourth model. Two-sided 5\% significance level was used, unless otherwise specified. Statistical procedures were carried out with SPSS (version 24.0, SPSS Inc, Chicago, IL, USA) and SAS Enterprise Guide 7.1.

\section{Results}

\section{Comparison of white matter characteristics between lesion timing groups}

Descriptives of each white matter characteristic of each tract can be found in Supplementary Material 3. Figure 2 presents FA images of a child with a PWM lesion and a child with a CDGM lesion. No significant differences in diffusion metrics were found for the CST, medial lemniscus or superior thalamic radiations of the less affected hemisphere ( $p>0.26)$, nor for the sensorimotor transcallosal fibers $(p>0.55)$ between children with PWM and CDGM lesions. In the lesioned hemisphere, mean diffusivity of the CST $(p=0.001$, and medial lemniscus ( $p=0.006$ ) was significantly higher in the CDGM group compared to the PWM group. Regarding the asymmetry indices, significantly more asymmetry was found for mean diffusivity of the CST ( $p=0.02$ ) and medial lemniscus $(p=0.049)$ in the CDGM group compared to the PWM group. No significant differences were found for the other asymmetry indices $(p>0.17)$.

\section{[Insert Figure 2 here]}

Fig. 2. FA images of three coronal slices of a child with a left PWM lesion (A, B and C) and a child with a right CDGM lesion (D, E and F).

\section{Comparison of white matter characteristics between CST wiring patterns}

Descriptives of each white matter characteristic of each tract can be found in Supplementary Material 4. Figure 3 displays the CST wiring patterns in two children categorized in the ipsilaterally wired group, one in the bilateral group and one in the contralateral group.

\section{[Insert Figure 3 here]}

Fig. 3. Corticospinal tracts in two ipsilaterally wired children (A, B and C, D), a bilaterally wired (E, F) and a contralaterally wired $(\mathrm{G}, \mathrm{H})$ child. Yellow tracts represent the tracts in the less affected hemisphere, blue tracts the lesioned hemisphere.

In the less affected hemisphere, only fractional anisotropy of the medial lemniscus was significantly different ( $p=0.002)$ between children with different CST wiring patterns. Post-hoc tests showed higher values in the 
contralaterally wired group as opposed to the ipsilaterally $(p=0.002)$ and bilaterally $(p=0.04)$ wired group. In the lesioned hemisphere, fractional anisotropy of the CST $(p=0.025)$ differed significantly between groups, indicating higher values in the contralaterally compared to the ipsilaterally wired group $(p=0.02)$. No significant differences were found for the diffusion metrics of the lesioned medial lemniscus and superior thalamic radiations, nor for the sensorimotor transcallosal fibers $(p>0.07)$. Regarding the asymmetry indices, the asymmetry index of streamline count of the CST differed significantly between groups $(p=0.005)$. Posthoc analyses revealed significantly less asymmetry in the contralateral compared to the ipsilateral $(p=0.002)$ and bilateral group $(p=0.02)$. A significant difference was also found for mean diffusivity of the medial lemniscus $(p=0.04)$. However, post-hoc differences did not reach statistical significance after Bonferroni correction $(p=0.07)$.

\section{Association between diffusion metrics and sensorimotor impairments}

Correlation coefficients between sensorimotor impairments and both diffusion metrics and asymmetry indices are displayed in Table 1. Moderate correlations were found between the motor impairments (i.e. muscle tone, muscle strength, grip strength) and all diffusion metrics of the CST ( $r_{b}=-0.49$ to $r_{s}=-0.69$ ), with strongest correlations for the streamline count asymmetry index $\left(r_{s}=-0.57\right.$ to -0.69$)$. For the sensory tracts, mean diffusivity of the medial lemniscus and the mean diffusivity asymmetry index of the medial lemniscus and superior thalamic radiations were also moderately correlated with the motor impairments $\left(r_{s}=-0.46\right.$ to $\left.r_{b}=-0.53\right)$. Regarding the sensorimotor transcallosal fibers, only one moderate correlation was found between mean diffusivity and muscle tone $\left(r_{p}=0.45\right)$.

For the sensory impairments (i.e. stereognosis and two-point discrimination), moderate correlations were found with mean diffusivity of the CST and its asymmetry index ( $r=-0.49$ to $r=-0.59$ ). For the medial lemniscus, moderate correlations were found between two-point discrimination and stereognosis with fractional anisotropy and mean diffusivity $(r=-0.50$ to -0.53$)$ as well as the mean diffusivity asymmetry index $\left(r_{b}=0.50\right.$ to 0.56 ). For the superior thalamic radiations, fractional anisotropy was moderately correlated with two-point discrimination $(r=0.50)$ and with stereognosis $(r=0.49)$. A moderate correlation was further found between the asymmetry index of mean diffusivity and two-point discrimination. Low to no correlations were found between the diffusion metrics of the sensorimotor transcallosal fibers and sensory impairments $\left(r_{s}=<0.36\right)$. 
Table 1. Correlation coefficients between diffusion metrics and sensorimotor outcomes.

\begin{tabular}{|c|c|c|c|c|c|}
\hline & Muscle tone & $\begin{array}{l}\text { Muscle } \\
\text { strength }\end{array}$ & Grip force & $2 \mathrm{PD}$ & Stereognosis \\
\hline \multicolumn{6}{|c|}{ Corticospinal tract } \\
\hline \multirow{2}{*}{$\mathrm{FA}_{\mathrm{L}}$} & $r=-0.33$ & $\mathrm{r}=0.42$ & $\mathrm{r}=0.44$ & - & $\mathrm{r}=0.23$ \\
\hline & $\mathrm{p}=0.09$ & $\mathrm{p}=0.024$ & $\mathrm{p}=0.018$ & & $\mathrm{p}=0.24$ \\
\hline \multirow{2}{*}{$\mathrm{MD}_{\mathrm{L}}$} & $r=0.49 *$ & $\mathrm{r}=-0.61 * *$ & $\mathrm{r}=-0.41$ & $\mathrm{r}=-\mathbf{0 . 4 9} *$ & $\mathbf{r}=-0.59 * *$ \\
\hline & $p=0.008$ & $p<0.001$ & $\mathrm{p}=0.026$ & $p=0.009$ & $p=0.001$ \\
\hline \multirow{2}{*}{ AI SC } & $r_{b}=-0.60 * *$ & $r_{s}=-0.57 *$ & $r_{s}=-0.69 * * *$ & - & $\mathrm{r}_{\mathrm{s}}=-0.35$ \\
\hline & $p=0.001$ & $p=0.001$ & $p<0.001$ & & $\mathrm{p}=0.07$ \\
\hline \multirow{2}{*}{ AI FA } & $r_{p}=-0.32$ & $r_{b}=-0.25$ & $\mathrm{r}_{\mathrm{b}}=-0.37$ & $\mathrm{r}_{\mathrm{b}}=0.23$ & - \\
\hline & $\mathrm{p}=0.09$ & $\mathrm{p}=0.19$ & $\mathrm{p}=0.05$ & $\mathrm{p}=0.24$ & \\
\hline \multirow{2}{*}{ AI MD } & $r_{b}=-0.49 *$ & $\mathbf{r}_{\mathrm{s}}=0.59 * *$ & $\mathrm{r}_{\mathrm{s}}=0.35$ & $\mathrm{r}_{\mathrm{s}}=0.41$ & $\mathbf{r}_{\mathrm{s}}=\mathbf{0 . 5 0} *$ \\
\hline & $p=0.007$ & $\mathrm{p}=0.001$ & $\mathrm{p}=0.06$ & $\mathrm{p}=0.03$ & $p=0.005$ \\
\hline \multicolumn{6}{|c|}{ Medial lemniscus } \\
\hline \multirow{2}{*}{$\mathrm{FA}_{\mathrm{L}}$} & $r=-0.27$ & $\mathrm{r}=0.32$ & $\mathrm{r}=0.33$ & $\mathrm{r}=0.52 *$ & $\mathrm{r}=0.52 *$ \\
\hline & $\mathrm{p}=0.14$ & $\mathrm{p}=0.08$ & $\mathrm{p}=0.07$ & $p=0.003$ & $p=0.003$ \\
\hline \multirow{2}{*}{$\mathrm{MD}_{\mathrm{L}}$} & $\mathrm{r}=0.51 *$ & $r_{s}=-0.46^{*}$ & $r=-0.46 *$ & $r_{s}=-0.50 *$ & $r=-0.53 *$ \\
\hline & $p=0.004$ & $\mathrm{p}=\mathbf{0 . 0 1}$ & $p=0.01$ & $p=0.005$ & $p=0.003$ \\
\hline AI SC & - & - & - & - & $\begin{array}{l}\mathrm{r}_{\mathrm{b}}=-0.32 \\
\mathrm{p}=0.08\end{array}$ \\
\hline \multirow{2}{*}{ AI FA } & - & $r_{s}=--0.27$ & _ & $\mathrm{r}_{\mathrm{s}}=-0.25$ & $\mathrm{r}_{\mathrm{s}}=-0.38$ \\
\hline & - & $\mathrm{p}=0.14$ & - & $\mathrm{p}=0.18$ & $\mathrm{p}=0.04$ \\
\hline \multirow{2}{*}{ AI MD } & $r_{p}=-0.53^{*}$ & $r_{b}=0.47 *$ & $r_{b}=0.48 *$ & $\mathbf{r}_{b}=0.50 *$ & $r_{b}=0.56 *$ \\
\hline & $p=0.002$ & $p=0.007$ & $p=0.005$ & $p=0.004$ & $\mathrm{p}=0.001$ \\
\hline \multicolumn{6}{|c|}{ Superior thalamic radiations } \\
\hline \multirow{2}{*}{$\mathrm{FA}_{\mathrm{L}}$} & $r=-0.33$ & $\mathrm{r}=0.47 *$ & $\mathrm{r}=0.33$ & $\mathbf{r}=\mathbf{0 . 5 0} *$ & $\mathrm{r}=0.49 *$ \\
\hline & $\mathrm{p}=0.09$ & $\mathrm{p}=0.01$ & $\mathrm{p}=0.08$ & $p=0.007$ & $p=0.009$ \\
\hline \multirow{2}{*}{$\mathrm{MD}_{\mathrm{L}}$} & $\mathrm{r}=0.35$ & $r=-0.40$ & $\mathrm{r}=-0.35$ & $\mathrm{r}=-0.41$ & $r=-0.28$ \\
\hline & $\mathrm{p}=0.07$ & $\mathrm{p}=0.03$ & $\mathrm{p}=0.06$ & $\mathrm{p}=0.03$ & $\mathrm{p}=0.15$ \\
\hline \multirow{2}{*}{ AI SC } & $\mathrm{r}_{\mathrm{b}}=0.26$ & $r_{s}=-0.33$ & - & - & - \\
\hline & $\mathrm{p}=0.17$ & $\mathrm{p}=0.08$ & - & - & - \\
\hline \multirow{2}{*}{ AI FA } & $r_{p}=0.26$ & $r_{b}=-0.39$ & - & $r_{b}=-0.36$ & $r_{b}=-0.32$ \\
\hline & $\mathrm{p}=0.17$ & $\mathrm{p}=0.03$ & & $\mathrm{p}=0.06$ & $\mathrm{p}=0.09$ \\
\hline \multirow{2}{*}{ AI MD } & $r_{p}=-0.49 *$ & $r_{b}=0.44$ & $\mathrm{r}_{\mathrm{b}}=0.41$ & $\mathbf{r}_{b}=0.53 *$ & $\mathrm{r}_{\mathrm{b}}=0.37$ \\
\hline & $\mathbf{p}=\mathbf{0 . 0 0 7}$ & $\mathrm{p}=0.016$ & $\mathrm{p}=0.02$ & $p=0.003$ & $\mathrm{p}=0.05$ \\
\hline \multicolumn{6}{|c|}{ Sensorimotor transcallosal fibers } \\
\hline \multirow[t]{2}{*}{ FA } & $r=-0.29$ & - & $\mathrm{r}=0.27$ & - & $\mathrm{r}=0.36$ \\
\hline & $\mathrm{p}=0.11$ & & $\mathrm{p}=0.13$ & 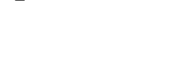 & $\mathrm{p}=0.045$ \\
\hline \multirow{2}{*}{ MD } & $\mathrm{r}=0.45 *$ & $\mathrm{r}=-0.36$ & $\mathrm{r}=-0.35$ & - & $\mathrm{r}=-0.23$ \\
\hline & $\mathrm{p}=\mathbf{0 . 0 1}$ & $\mathrm{p}=0.042$ & $\mathrm{p}=0.043$ & & $\mathrm{p}=0.2$ \\
\hline
\end{tabular}

2PD, two-point discrimination; SC, streamline count; FA, fractional anisotropy; MD, mean diffusivity; L, lesioned hemisphere; AI, asymmetry index; $r$, partial correlations; $r_{p}$, pearson correlation; $r_{b}$, biserial correlation; $r_{s}$, spearman correlation; -, no correlation $(r<0.21) ; 0.21-0.39$, 
Table 2. Correlation coefficients between diffusion metrics and activity outcomes.

\begin{tabular}{|c|c|c|c|c|c|c|c|}
\hline & AHA & MA2 ROM & MA2 ACC & MA2 DEX & MA2 FL & JTHFT & ABILHAND \\
\hline \multicolumn{8}{|c|}{ Corticospinal tract } \\
\hline \multirow{2}{*}{$\mathrm{FA}_{\mathrm{L}}$} & $\mathrm{r}=0.38$ & $\mathrm{r}=0.24$ & - & - & $\mathrm{r}=0.32$ & $\mathrm{r}=0.34$ & $\mathrm{r}=0.26$ \\
\hline & $\mathrm{p}=0.044$ & $\mathrm{p}=0.20$ & & & $\mathrm{p}=0.09$ & $\mathrm{p}=0.07$ & $\mathrm{p}=0.17$ \\
\hline \multirow{2}{*}{$\mathrm{MD}_{\mathrm{L}}$} & $r=-0.56 *$ & $\mathbf{r}=-0.62 * *$ & $\mathrm{r}=-0.37$ & $\mathrm{r}=-0.62 * *$ & $\mathrm{r}=-0.32$ & $r=-0.48 *$ & $r=-0.39$ \\
\hline & $p=0.002$ & $\mathrm{p}<0.001$ & $\mathrm{p}=0.05$ & $\mathrm{p}<0.001$ & $\mathrm{p}=0.09$ & $p=0.008$ & $\mathrm{p}=0.04$ \\
\hline \multirow{2}{*}{ AI SC } & $\mathbf{r}_{\mathbf{b}}=-\mathbf{0 . 7 2} * * *$ & $r_{s}=-0.51 *$ & - & $r_{b}=-0.57 *$ & $r_{s}=-0.57 *$ & $r_{s}=-0.55 *$ & $\mathrm{r}_{\mathrm{s}}=-0.34$ \\
\hline & $\mathrm{p}<0.0001$ & $p=0.004$ & & $p=0.001$ & $p=0.001$ & $p=0.002$ & $\mathrm{p}=0.07$ \\
\hline \multirow{2}{*}{ AI FA } & $r_{p}=-0.23$ & - & $\mathrm{r}_{\mathrm{b}}=0.28$ & - & $\mathrm{r}_{\mathrm{b}}=-0.29$ & $\mathrm{r}_{\mathrm{b}}=-0.21$ & - \\
\hline & $\mathrm{p}=0.21$ & & $\mathrm{p}=0.13$ & & $\mathrm{p}=0.13$ & $\mathrm{p}=0.26$ & \\
\hline \multirow{2}{*}{ AI MD } & $r_{b}=-0.49 *$ & $r_{\mathrm{s}}=0.45 *$ & - & $r_{b}=0.45^{*}$ & - & $\mathrm{r}_{\mathrm{s}}=0.32$ & $r_{s}=0.36$ \\
\hline & $p=0.006$ & $p=0.01$ & & $\mathrm{p}=0.01$ & & $\mathrm{p}=0.08$ & $\mathrm{p}=0.05$ \\
\hline \multicolumn{8}{|c|}{ Medial lemniscus } \\
\hline \multirow{2}{*}{$\mathrm{FA}_{\mathrm{L}}$} & $\mathrm{r}=0.37$ & $\mathrm{r}=0.25$ & - & $\mathrm{r}=0.27$ & - & $\mathrm{r}=0.38$ & $\mathrm{r}=0.25$ \\
\hline & $\mathrm{p}=0.04$ & $\mathrm{p}=0.17$ & & $\mathrm{p}=0.14$ & & $\mathrm{p}=0.04$ & $\mathrm{p}=0.18$ \\
\hline \multirow{2}{*}{$\mathrm{MD}_{\mathrm{L}}$} & $\mathbf{r}=-0.62 * *$ & $\mathbf{r}=-0.62 * *$ & $\mathrm{r}=-0.34$ & $\mathbf{r}=-0.67 * * *$ & $\mathrm{r}=-0.37$ & $\mathbf{r}=-0.60 * *$ & $r=-0.37$ \\
\hline & $\mathrm{p}<0.001$ & $\mathrm{p}<0.001$ & $\mathrm{p}=0.07$ & $\mathrm{p}<0.0001$ & $\mathrm{p}=0.04$ & $\mathrm{p}<0.001$ & $\mathrm{p}=0.04$ \\
\hline AI SC & - & - & - & - & - & - & - \\
\hline \multirow[t]{2}{*}{ AI FA } & $r_{b}=-0.26$ & $r_{s}=-0.32$ & - & $\mathrm{r}_{\mathrm{b}}=-0.24$ & $r_{s}=-0.37$ & $r_{s}=-0.37$ & - \\
\hline & $\mathrm{p}=0.16$ & $\mathrm{p}=0.07$ & & $\mathrm{p}=0.18$ & $\mathrm{p}=0.04$ & $\mathrm{p}=0.04$ & \\
\hline \multirow{2}{*}{ AI MD } & $\mathbf{r}_{b}=0.60 * *$ & $r_{b}=0.51 *$ & $\mathrm{r}_{\mathrm{b}}=0.24$ & $r_{p}=0.60 * *$ & $\mathrm{r}_{\mathrm{b}}=0.32$ & $\mathbf{r}_{b}=0.62 * *$ & $\mathrm{r}_{\mathrm{b}}=0.33$ \\
\hline & $\mathrm{p}<0.001$ & $p=0.003$ & $\mathrm{p}=0.18$ & $\mathrm{p}<0.001$ & $\mathrm{p}=0.08$ & $\mathrm{p}<0.001$ & $\mathrm{p}=0.07$ \\
\hline \multicolumn{8}{|c|}{ Superior thalamic radiations } \\
\hline \multirow{2}{*}{$\mathrm{FA}_{\mathrm{L}}$} & $r=0.43$ & $\mathrm{r}=0.41$ & - & $\mathrm{r}=0.23$ & $\mathrm{r}=0.33$ & $\mathrm{r}=0.40$ & $\mathrm{r}=0.28$ \\
\hline & $\mathrm{p}=0.02$ & $\mathrm{p}=0.03$ & & $\mathrm{p}=0.24$ & $\mathrm{p}=0.08$ & $\mathrm{p}=0.03$ & $\mathrm{p}=0.15$ \\
\hline \multirow{2}{*}{$\mathrm{MD}_{\mathrm{L}}$} & $r=-0.49 *$ & $r=-0.51 *$ & $\mathrm{r}=-0.40$ & $\mathrm{r}=-0.59 * *$ & $\mathrm{r}=-0.43$ & $\mathrm{r}=-0.51 *$ & $\mathrm{r}=-0.30$ \\
\hline & $\mathrm{p}=\mathbf{0 . 0 0 8}$ & $p=0.005$ & $\mathrm{p}=0.03$ & $\mathrm{p}=\mathbf{0 . 0 0 1}$ & $\mathrm{p}=0.02$ & $p=0.005$ & $\mathrm{p}=0.11$ \\
\hline \multirow[t]{2}{*}{ AI SC } & $\mathrm{r}_{\mathrm{b}}=-0.21$ & - & - & - & - & - & - \\
\hline & $\mathrm{p}=0.27$ & & & & & & \\
\hline \multirow{3}{*}{ AI FA } & $r_{p}=-0.30$ & $r_{b}=-0.42$ & - & - & $r_{b}=-0.35$ & - & $r_{b}=-0.24$ \\
\hline & $\mathrm{p}=0.11$ & $\mathrm{p}=0.02$ & & & $\mathrm{p}=0.06$ & & $\mathrm{p}=0.20$ \\
\hline & $r_{p}=0.51 *$ & $r_{b}=0.44$ & $\mathrm{r}_{\mathrm{b}}=0.26$ & $r_{p}=0.52 *$ & $\mathrm{r}_{\mathrm{b}}=0.39$ & $r_{b}=0.56 *$ & $\mathrm{r}_{\mathrm{b}}=0.30$ \\
\hline
\end{tabular}




\begin{tabular}{|c|c|c|c|c|c|c|c|}
\hline AI MD & $p=0.004$ & $\mathrm{p}=0.015$ & $\mathrm{p}=0.17$ & $p=0.003$ & $\mathrm{p}=0.03$ & $p=0.001$ & $\mathrm{p}=0.11$ \\
\hline \multicolumn{8}{|c|}{ Sensorimotor transcallosal fibers } \\
\hline \multirow{2}{*}{ FA } & $\mathrm{r}=0.38$ & $\mathrm{r}=0.34$ & $\mathrm{r}=0.39$ & $\mathrm{r}=0.34$ & $\mathrm{r}=0.43$ & $\mathrm{r}=0.35$ & $r=0.27$ \\
\hline & $\mathrm{p}=0.03$ & $\mathrm{p}=0.05$ & $\mathrm{p}=0.03$ & $\mathrm{p}=0.05$ & $\mathrm{p}=0.01$ & $\mathrm{p}=0.05$ & $\mathrm{p}=0.14$ \\
\hline \multirow{2}{*}{ MD } & $\mathrm{r}=-0.47 *$ & $r=-0.46 *$ & $\mathrm{r}=-0.32$ & $\mathrm{r}=-0.42$ & $r=-0.29$ & $r=-0.45 *$ & $\mathrm{r}=-0.29$ \\
\hline & $\mathrm{p}=0.006$ & $\mathbf{p}=\mathbf{0 . 0 0 7}$ & $\mathrm{p}=0.07$ & $\mathrm{p}=0.015$ & $\mathrm{p}=0.1$ & $p=0.008$ & $\mathrm{p}=0.1$ \\
\hline
\end{tabular}

FA, fractional anisotropy; $\mathrm{MD}$, mean diffusivity; $\mathrm{L}$, lesioned hemisphere; $\mathrm{AI}$, asymmetry index; $r$, partial correlations; $r_{p}$ pearson correlation; $r_{b}$, biserial correlation; $r_{s}$, spearman correlation; -, no correlation $(r<0.21) ; 0.21-0.39$, low correlation; 0.40-0.69, moderate correlation; 0.70-0.89; high correlation; ***, $\mathrm{p}<0.0001 ; * *, \mathrm{p}<0.001 ; *, \mathrm{p}<0.01$. 


\section{Association between diffusion metrics and activity limitations}

Correlation coefficients between activity limitations and both diffusion metrics and asymmetry indices are summarized in Table 2. Mostly moderate correlations were found for the CST and the AHA $\left(r_{p}=0.49\right.$ to $r=-0.56$ ), the MA2 ( $r_{b}=0.45$ to $r=-0.67$ ) and the JTHFT ( $r=-0.48$ to $r_{s}=-0.55$ ). The streamline count asymmetry index of the CST was highly correlated with the AHA $\left(r_{b}=0.72\right)$. For both sensory tracts, only mean diffusivity and its asymmetry index were moderately correlated with the AHA ( $r=-0.49$ to $r=-0.62$ ), the MA2 (ROM and dexterity subscales; $r=-0.51$ to $r=-0.67$ ) and the JTHFT ( $r=-0.51$ to $r_{b}=-$ 0.62). For the sensorimotor transcallosal fibers, mean diffusivity was related with the AHA $(r=-0.47)$, the MA2 ROM subscale $\left(r_{b}=-0.46\right)$ and the JTHFT $(r=-0.45)$. Only low correlations were found for the ABILHAND-kids questionnaire $(r<-0.39)$.

\section{Multiple regression}

For these analyses, only children with TMS-data available were included $(\mathrm{N}=30)$.

The output of the four models is summarized in Table 3 for both outcome measures. Each of the four models could significantly explain bimanual performance $(\mathrm{p}<0.002)$ and unimanual capacity $(\mathrm{p}<0.02)$. Model 1 explained 50\% of the variance in bimanual performance by solely diffusion metrics of the sensory tracts $(\mathrm{p}=0.002)$, while $43 \%$ of the variance in unimanual capacity was explained by both the sensory tracts and the CST $(\mathrm{p}=0.02)$. Model 2 revealed that the variance in bimanual performance $(74 \%$, $\mathrm{p}<0.0001)$ and unimanual capacity $(50 \%, \mathrm{p}=0.004)$ was explained by a combination of the asymmetry indices of the CST and sensory tracts. In Model 3 and Model 4, lesion timing and type of the CST wiring pattern were added to the independent variables of Model 1 and Model 2, respectively. In Model 3, the type of CST wiring pattern was retained along with diffusion metrics of the sensory tracts and the CST explaining $62 \%$ of the variance in bimanual performance $(\mathrm{p}=0.0001)$ and $41 \%$ of unimanual capacity $(\mathrm{p}=0.01$ ). In contrast, in Model 4, when using the asymmetry indices, type of CST wiring pattern was not retained in both outcome measures. However, lesion timing contributed significantly in explaining unimanual capacity $(58 \%, \mathrm{p}=0.0002)$." 
Table 3. Four regression models explaining bimanual performance or unimanual capacity

Table 3A. R ${ }^{2}$ Squared Selection Method explaining bimanual performance

\begin{tabular}{|c|c|c|c|c|c|c|c|c|c|c|c|}
\hline \multicolumn{3}{|c|}{ Model 1} & \multicolumn{3}{|c|}{ Model 2} & \multicolumn{3}{|c|}{ Model 3} & \multicolumn{3}{|c|}{ Model 4} \\
\hline Best set & $\mathbf{R}^{2}$ & p-value & Best set & $\mathbf{R}^{2}$ & p-value & Best set & $\mathbf{R}^{2}$ & p-value & Best set & $\mathbf{R}^{2}$ & p-value \\
\hline MD ML & $38 \%$ & 0.0009 & $\begin{array}{c}\text { SC AI } \\
\text { CST }\end{array}$ & $53 \%$ & $<0.0001$ & $\begin{array}{c}\text { CST } \\
\text { wiring }\end{array}$ & $42 \%$ & 0.0005 & $\begin{array}{l}\text { SC AI } \\
\text { CST }\end{array}$ & $53 \%$ & $<0.0001$ \\
\hline FA STR & $11 \%$ & 0.04 & $\begin{array}{c}\text { FA AI } \\
\text { CST }\end{array}$ & $11 \%$ & 0.01 & MD ML & $15 \%$ & 0.01 & $\begin{array}{l}\text { FA AI } \\
\text { CST }\end{array}$ & $11 \%$ & 0.01 \\
\hline $\begin{array}{l}\text { MD } \\
\text { STR }\end{array}$ & $1 \%$ & 0.88 & $\begin{array}{c}\text { MD AI } \\
\text { ML }\end{array}$ & $10 \%$ & 0.02 & FA STR & $5 \%$ & 0.12 & $\begin{array}{l}\text { MD AI } \\
\text { ML }\end{array}$ & $10 \%$ & 0.02 \\
\hline $\begin{array}{c}\text { Full } \\
\text { model }\end{array}$ & $50 \%$ & 0.002 & $\begin{array}{c}\text { Full } \\
\text { model }\end{array}$ & $74 \%$ & $<0.0001$ & $\begin{array}{c}\text { Full } \\
\text { model }\end{array}$ & $62 \%$ & 0.0001 & $\begin{array}{l}\text { Full } \\
\text { model }\end{array}$ & $74 \%$ & $<0.0001$ \\
\hline
\end{tabular}

Table 3B. R $^{2}$ Squared Selection Method explaining unimanual capacity

\begin{tabular}{|c|c|c|c|c|c|c|c|c|c|c|c|}
\hline \multicolumn{3}{|c|}{ Model 1} & \multicolumn{3}{|c|}{ Model 2} & \multicolumn{3}{|c|}{ Model 3} & \multicolumn{3}{|c|}{ Model 4} \\
\hline Best set & $\mathbf{R}^{2}$ & p-value & Best set & $\mathbf{R}^{2}$ & p-value & Best set & $\mathbf{R}^{2}$ & p-value & Best set & $\mathbf{R}^{2}$ & p-value \\
\hline FA STR & $36 \%$ & 0.003 & $\begin{array}{l}\text { FA AI } \\
\text { CST }\end{array}$ & $26 \%$ & 0.005 & FA STR & $32 \%$ & 0.005 & $\begin{array}{c}\text { FA AI } \\
\text { CST }\end{array}$ & $31 \%$ & 0.001 \\
\hline $\begin{array}{l}\text { MD } \\
\text { CST }\end{array}$ & $6 \%$ & 0.17 & $\begin{array}{l}\text { SC AI } \\
\text { CST }\end{array}$ & $21 \%$ & 0.03 & $\begin{array}{c}\text { CST } \\
\text { wiring }\end{array}$ & $6 \%$ & 0.16 & $\begin{array}{l}\text { SC AI } \\
\text { CST }\end{array}$ & $17 \%$ & 0.04 \\
\hline MD ML & $1 \%$ & 0.58 & $\begin{array}{c}\text { MD AI } \\
\text { STR }\end{array}$ & $\%$ & & $\begin{array}{l}\text { MD } \\
\text { CST }\end{array}$ & $3 \%$ & 0.34 & $\begin{array}{l}\text { Lesion } \\
\text { timing }\end{array}$ & $10 \%$ & 0.03 \\
\hline $\begin{array}{c}\text { Full } \\
\text { model }\end{array}$ & $43 \%$ & 0.02 & $\begin{array}{c}\text { Full } \\
\text { model }\end{array}$ & $50 \%$ & 0.004 & $\begin{array}{c}\text { Full } \\
\text { model }\end{array}$ & $41 \%$ & 0.01 & $\begin{array}{c}\text { Full } \\
\text { model }\end{array}$ & $58 \%$ & 0.0002 \\
\hline
\end{tabular}

\section{Discussion}

This study compared white matter characteristics of the main motor tract (CST), two sensory tracts (medial lemniscus, superior thalamic radiations) and the sensorimotor transcallosal fibers between different classification groups (i.e. lesion timing and CST wiring pattern) in 34 children with unilateral CP. We also gained better understanding of how white matter characteristics of each of these tracts relate to different aspects of upper limb function. Moreover, we demonstrated the importance of taking into account the integrity of both motor and sensory tracts of both hemispheres to explain the variability in upper limb function in children with unilateral CP. 
In damaged white matter, fractional anisotropy typically decreases as a result of coherence loss in the main preferred diffusion direction, while mean diffusivity increases due to increased free diffusion (Soares et al. 2013). In this study, we investigated for the first time whether there are differences in white matter characteristics between children with different lesion timings as well as between children with different CST wiring patterns. The study results showed for the first time that the CST and medial lemniscus are more severely damaged in children with CDGM lesions compared to children with PWM lesions, as shown by higher values of mean diffusivity in the CDGM group. A significantly more damaged CST was also found in the ipsilaterally compared to the contralaterally wired group. Children with an ipsilateral CST wiring pattern exhibited lower fractional anisotropy values and more asymmetry in streamline count between both CST's. Eyre et al. (2007) have indeed reported previously that an ipsilateral CST wiring pattern results from a gradual weakening of the contralateral CST projections and strengthening of the ipsilateral CST projections.

Surprisingly, also fractional anisotropy of the medial lemniscus in the less affected hemisphere was significantly lower in the ipsilaterally and bilaterally wired group compared to the contralaterally wired group. Studies using conventional brain imaging techniques reported bilateral brain lesions in up to 50\% of children with unilateral CP (Holmström et al. 2010; Feys et al. 2010). However, DTI has been shown to be more accurate in detecting white matter abnormalities (Son et al. 2007). Also Scheck et al. (2014) reported bilaterally reduced thalamic volumes in children with apparent unilateral lesions on conventional imaging using voxel-based morphometry. Hence, more sensitive measures to assess the integrity of the less affected hemisphere in children with unilateral CP are clearly needed. The importance of this clarification lies within the recent findings of a discrete decrease in performance of the dominant hand in children with unilateral CP compared to typically developing children (Rich et al. 2017; Basu et al. 2017).

Secondly, we found that increased muscle tone and weakness were moderately associated with a more damaged CST and medial lemniscus. The CST is the main motor pathway for voluntary motor control (Porter and Lemon 1993), but also sensory feedback is indispensable for adequate motor function (Perruchoud et al. 2014; Gordon, Charles, and Steenbergen 2006). To the best of our knowledge, these relationships have not been described previously, causing this study to be the first to report on the relationship between white matter characteristics of motor and sensory tracts with motor impairments in children with unilateral CP. We further found a relationship between more severe sensory impairments and increased damage of the medial lemniscus and superior thalamic radiation, which corresponds to previous studies (Tsao et al. 2013; Kuczynski et al. 2017; Weinstein et al. 2018). 
This study further showed that a more impaired bimanual performance and unimanual capacity was related with more damage to both motor (Holmström et al. 2011; Pannek et al. 2014; Tsao et al. 2014; Scheck et al. 2016; Hodge et al. 2017; Gupta et al. 2017) and sensory tracts (Rose et al. 2011; Pannek et al. 2014; Tsao et al. 2013; Gupta et al. 2017). Although correlations were lower, bimanual performance and unimanual capacity were also correlated with white matter characteristics of the sensorimotor transcallosal fibers, which is in line with Weinstein et al. $(2014,2018)$. In contrast, no relationship was found with the sensorimotor impairments, pointing towards a more important role of interhemispheric communication in the execution of more complex upper limb tasks (Roebuck-Spencer et al. 2004; Gooijers and Swinnen 2014).

Finally, regression analysis revealed that $74 \%$ of the variance in bimanual performance and $50 \%$ of the variance in unimanual capacity could be explained by the asymmetry indices of the CST and sensory tracts (i.e. medial lemniscus and superior thalamic radiation, respectively). To the best of our knowledge, this is the first time such a high amount of explained variance could be revealed by combining multiple white matter tracts, which is a unique contribution to the existing literature. Nevertheless, Gupta et al. (2017) suggested a more significant contribution of the medial lemniscus to upper limb motor and sensory function compared to the CST. Yet, Gupta et al. did not consider the severity of the white matter damage, as they used dichotomous scores (i.e. tract present or absent). Hence, this suggests that continuous variables reflecting the severity of white matter damage may be important when predicting upper limb function in children with unilateral CP. Nevertheless, previous (Pannek et al. 2014; Gupta et al. 2017) and current study results underline the significance of both motor and sensory tracts for bimanual performance and unimanual capacity highlighting the necessity of preserved sensorimotor integration.

Overall, the models explaining bimanual performance were stronger compared to unimanual capacity. In particular the asymmetry indices explain a much higher amount of variance in bimanual performance compared to only the diffusion metrics of the lesioned hemisphere ( $74 \%$ versus $50 \%$, respectively), while for unimanual capacity, both models explain more similar percentages (50\% versus 43\%, respectively). This finding is a novel contribution to the existing literature and warrants further exploration.

The high amount of explained variance in bimanual performance by the asymmetry indices underlines the relevance of considering both hemispheres despite the unilateral clinical presentation. Asymmetry indices may also be better determinants as they may take potential reorganization mechanisms in the less affected hemisphere into account (Pagnozzi et al. 2016), possibly explaining why the type of CST wiring pattern was not retained in Model 4 next to the asymmetry indices. This finding further points towards a promising predictive value of the asymmetry indices of the CST, being a potential alternative 
for TMS. Future studies are needed to investigate to what extent asymmetry indices of the CST are a reliable substitute for the type of CST wiring pattern assessed with TMS. Nevertheless, our results suggest that the type of CST wiring pattern is the strongest determinant of bimanual performance, either measured directly with TMS (i.e. model 3) or indirectly with the asymmetry indices (i.e. model 4). This is in agreement with the recent findings of Simon-Martinez et al. (2018), who also reported that the type of CST wiring pattern was the strongest predictor of bimanual performance. For unimanual capacity, the type of CST wiring pattern seems to play a less significant role. Together, these results imply that children with ipsilateral CST projections may still develop a functional grasp function (Staudt et al. 2002), yet the integration of using both hands together may be more severely compromised.

Interestingly, for unimanual capacity, lesion timing contributed significantly to the explained variance along with the asymmetry indices of the CST. A possible explanation for this finding is that in children with later lesions, sensory deficits are usually more pronounced (Feys et al. 2010; Mailleux et al. 2017; Simon-Martinez et al. 2018). Gupta et al. (2017) indeed showed that the sensory physiology of the medial lemniscus is crucial for performing unimanual movements. Nevertheless, large population based studies are crucial to confirm our findings and further unravel the relative importance of each of these neurological factors as well as their interactions.

Some critical reflections on this study are also necessary. First, we used a diffusion tensor analysis with deterministic tractography, despite the high $b$-value. A brief evaluation attempt was performed showing more stable results with DTI compared to single shell constrained spherical deconvolution, which made us choose for deterministic tractography to analyze the data. Despite the use of an upper threshold of $2.5 \mathrm{~mm}$ for motion during the scans and the use of REKINDLE for outlier detection and removal (Tax et al. 2015), outliers in the data may still be caused by sub-threshold motion or large brain lesions. Another discussion point is the manual ROI delineation. Manual ROI delineation is more subjective. However, it allows to take the neuroanatomical variability, inherent to the neuropathophysiology of the $\mathrm{CP}$ population into account. In this study sample, there is a large variability regarding age range (5y to $15 y$ ) as well as lesion severity (focal lesions to lesions extending over the whole hemisphere) which impacts brain anatomy and consequently hampers the use of templates/a priori labels (atlases). We made the ROI drawing as consistent as possible by focusing on specific, delineated landmarks such as the pons, PLIC, central sulcus and hand knob. In addition, dice coefficients of all fiber bundles were good, except for moderate coefficients for the superior thalamic radiation in the lesioned hemisphere. Thirdly, despite revealing significant differences, the relatively small subgroups (i.e. lesion timing and type of CST wiring pattern) may have resulted in insufficient power to reveal subtle differences in the less affected hemisphere. Also, no correction for multiple testing was applied due to the explorative nature of this research question. Moreover, four children with CDGM lesions were excluded due to their extended lesions and the inability to generate none of the targeted tracts. This has caused an unintentional 
selection bias reducing the amount of variability in this group, but also underlines the difficulty of applying tractography in this patient group. In the future, international collaborations are needed to obtain a higher number of participants in each subgroup to confirm our study findings. Next, no control group with typically developing children was included in this study. However, previous studies have already shown differences in DTI metrics between children with unilateral CP and typically developing children (Holmström et al. 2011; Hodge et al. 2017; Kuczynski et al. 2017; Pannek et al. 2014; Scheck et al. 2016; Thomas et al. 2005; Tsao et al. 2013; 2014; Weinstein et al. 2014). Finally, in seven of the eleven children classified in the ipsilateral CST wiring group, contralateral CST projections were found with tractography. However, it has been reported previously that the thresholds for activation of the lesioned CST can be high in children with unilateral CP (Eyre et al. 2007; Kuo et al. 2017; Basu 2017). On the other hand, if contralateral CST projections are reconstructed in ipsilaterally wired children, it may be that these contralateral projections are not functional. Future studies combining functional and diffusion MRI are warranted to aid in this clarification.

\section{Conclusion}

This study was the first to demonstrate differences in white matter characteristics between children with different lesion timings and different types of CST wiring patterns. Secondly, our results emphasized the importance of not only the motor pathways, but also the sensory and interhemispheric pathways for upper limb function. Remarkably, up to $74 \%$ of the variance in bimanual performance and up to $50 \%$ of the variance in unimanual capacity could be explained by the asymmetry indices of the CST and sensory tracts. Adding lesion timing and type of CST wiring pattern did not further improve the model of bimanual performance, while for unimanual capacity lesion timing was additionally retained. The results of this study contribute to a better understanding of the underlying neuropathology of upper limb function and point towards the importance of using tractography in the clinical setting for defining prognoses regarding upper limb functional outcomes in children with unilateral CP.

Funding This study was funded by the Fund Scientific Research Flanders (FWO project, grant G087213N), by the Special Research Fund, KU Leuven (grant OT/14/127).

Conflict of Interest The authors declare that they have no conflict of interest.

Informed consent Informed consent was obtained from all parents of all individual participants included in the study. Children older than 12 years were also asked for their assent.

Ethical approval All procedures performed in studies involving human participants were in accordance with the ethical standards of the institutional and/or national research committee and with the 1964 Helsinki declaration and its later amendments or comparable ethical standards. 


\section{References}

Ariola, Mariano M. 2006. Principles and Methods of Research. Rex Bookstore, Inc.

Arnould, Carlyne, Massimo Penta, Anne Renders, and Jean-louis Thonnard. 2004. "A Measure of Manual Ability in Children with Cerebral Palsy." Neurology 63 (5375): 1045-52. https://doi.org/10.1212/01.WNL.0000138423.77640.37.

Basu, Anna P, Emma V Kirkpatrick, Blythe Wright, Janice E Pearse, Kate E Best, and Janet A Eyre. 2017. "The Tyneside Pegboard Test: Development, Validation, and Observations in Unilateral Cerebral Palsy." Developmental Medicine \& Child Neurology, December. https://doi.org/10.1111/dmcn.13645.

Basu, Anna Purna. 2017. "Mapping Corticospinal Tract Projection Patterns in Unilateral Cerebral Palsy." Developmental Medicine and Child Neurology 59 (1): 10-11. https://doi.org/10.1111/dmen.13209.

Beckung, E., G. Hagberg, P. Uldall, and C. Cans. 2008. "Probability of Walking in Children With Cerebral Palsy in Europe." Pediatrics 121 (1): e187-92. https://doi.org/10.1542/peds.2007-0068.

Bohannon, Richard W, and Melissa B Smith. 1987. "Inter Rater Reliability of a Modified Ashworth Scale of Muscle Spasticity.” Phys Ther 67: 206-7. https://doi.org/10.1007/978-1-4471-5451$8 \_105$.

Colver, Allan, Charles Fairhurst, and Peter O D Pharoah. 2014. "Cerebral Palsy." The Lancet 383 (9924): 1240-49. https://doi.org/10.1016/S0140-6736(13)61835-8.

Dice, L. 1945. "Measures of the Amount of Ecologic Association between Species." Ecology 26: 297302.

Eyre, Janet A, Martin Smith, Lyvia Dabydeen, Gavin J Clowry, Eliza Petacchi, Roberta Battini, Andrea Guzzetta, and Giovanni Cioni. 2007. "Is Hemiplegic Cerebral Palsy Equivalent to Amblyopia of the Corticospinal System ?," 493-503. https://doi.org/10.1002/ana.21108.

Feys, Hilde, Maria Eyssen, Ellen Jaspers, Katrijn Klingels, Kaat Desloovere, Guy Molenaers, and Paul De Cock. 2010. "Relation between Neuroradiological Findings and Upper Limb Function in Hemiplegic Cerebral Palsy." European Journal of Paediatric Neurology 14 (2): 169-77. https://doi.org/10.1016/j.ejpn.2009.01.004.

Freund, Rudolf J., and Ramon C. Littell. 2000. SAS® System for Regression. Third edit. Cary, NC: SAS Institute Inc.

Gooijers, J., and S. P. Swinnen. 2014. "Interactions between Brain Structure and Behavior: The Corpus Callosum and Bimanual Coordination." Neuroscience and Biobehavioral Reviews 43: 119. https://doi.org/10.1016/j.neubiorev.2014.03.008.

Gordon, Andrew M., Jeanne Charles, and Bert Steenbergen. 2006. "Fingertip Force Planning during Grasp Is Disrupted by Impaired Sensorimotor Integration in Children with Hemiplegic Cerebral Palsy." Pediatric Research 60 (5): 587-91. https://doi.org/10.1203/01.pdr.0000242370.41469.74.

Gupta, Disha, Alexandre Barachant, Andrew M. Gordon, Claudio Ferre, Hsing Ching Kuo, Jason B. Carmel, and Kathleen M. Friel. 2017. "Effect of Sensory and Motor Connectivity on Hand Function in Pediatric Hemiplegia." Annals of Neurology 82 (5): 766-80. https://doi.org/10.1002/ana.25080.

Harrell, Frank E., Kerry L. Lee, and Daniel B. Mark. 2005. "Prognostic/Clinical Prediction Models: Multivariable Prognostic Models: Issues in Developing Models, Evaluating Assumptions and Adequacy, and Measuring and Reducing Errors." Tutorials in Biostatistics, Statistical Methods in Clinical Studies 1: 223-49. https://doi.org/10.1002/0470023678.ch2b(i).

Hoare, Brian, Michael Ditchfield, Megan Thorley, Margaret Wallen, Jenny Bracken, Adrienne Harvey, Catherine Elliott, Iona Novak, and Ali Crichton. 2018. "Cognition and Bimanual Performance in Children with Unilateral Cerebral Palsy : Protocol for a Multicentre, CrossSectional Study," 1-12.

Hodge, Jacquie, Bradley Goodyear, Helen Carlson, Xing Chang Wei, and Adam Kirton. 2017. "Segmental Diffusion Properties of the Corticospinal Tract and Motor Outcome in Hemiparetic Children with Perinatal Stroke." Journal of Child Neurology 32 (6): 550-59. https://doi.org/10.1177/0883073817696815.

Hofer, Sabine, and Jens Frahm. 2006. "Topography of the Human Corpus Callosum RevisitedComprehensive Fiber Tractography Using Diffusion Tensor Magnetic Resonance Imaging." 
NeuroImage 32 (3): 989-94. https://doi.org/10.1016/j.neuroimage.2006.05.044.

Holmefur, Marie M., and Lena Krumlinde-Sundholm. 2016. "Psychometric Properties of a Revised Version of the Assisting Hand Assessment (Kids-AHA 5.0)." Developmental Medicine and Child Neurology 58 (6): 618-24. https://doi.org/10.1111/dmcn.12939.

Holmström, Linda, F. Lennartsson, Ann-Christin Eliasson, Olof Flodmark, C. Clark, Kristina Tedroff, Hans Forssberg, and Brigitte Vollmer. 2011. "Diffusion MRI in Corticofugal Fibers Correlates with Hand Function in Unilateral Cerebral Palsy." Neurology 77 (8): 775-83. https://doi.org/10.1212/WNL.0b013e31822b0040.

Holmström, Linda, Brigitte Vollmer, Kristina Tedroff, Mominul Islam, Jonas K.E. Persson, Annika Kits, Hans Forssberg, and Ann Christin Eliasson. 2010. "Hand Function in Relation to Brain Lesions and Corticomotor-Projection Pattern in Children with Unilateral Cerebral Palsy." Developmental Medicine and Child Neurology 52 (2): 145-52. https://doi.org/10.1111/j.14698749.2009.03496.x.

Jones, Derek K., Thomas R. Knösche, and Robert Turner. 2013. "White Matter Integrity, Fiber Count, and Other Fallacies: The Do's and Don'ts of Diffusion MRI." NeuroImage 73: 239-54. https://doi.org/10.1016/j.neuroimage.2012.06.081.

Klingels, Katrijn, Paul De Cock, Guy Molenaers, Kaat Desloovere, Catherine Huenaerts, Ellen Jaspers, and Hilde Feys. 2010. "Upper Limb Motor and Sensory Impairments in Children with Hemiplegic Cerebral Palsy. Can They Be Measured Reliably?” Disabil Rehabil 32 (5): 409-16. https://doi.org/10.3109/09638280903171469.

Klingels, Katrijn, Inge Demeyere, Ellen Jaspers, Paul De Cock, Guy Molenaers, Roslyn Boyd, and Hilde Feys. 2012. "Upper Limb Impairments and Their Impact on Activity Measures in Children with Unilateral Cerebral Palsy." European Journal of Paediatric Neurology 16 (5): 475-84. https://doi.org/10.1016/j.ejpn.2011.12.008.

Krägeloh-Mann, Ingeborg, and Veronka Horber. 2007. "The Role of Magnetic Resonance Imaging in Elucidating the Pathogenesis of Cerebral Palsy: A Systematic Review." Developmental Medicine \& Child Neurology 49: 144-51. https://doi.org/10.1111/j.1469-8749.2007.00144.x.

Krumlinde-Sundholm, Lena, Marie Holmefur, Anders Kottorp, and Ann Christin Eliasson. 2007. "The Assisting Hand Assessment: Current Evidence of Validity, Reliability, and Responsiveness to Change." Developmental Medicine and Child Neurology 49 (4): 259-64. https://doi.org/10.1111/j.1469-8749.2007.00259.x.

Kuczynski, Andrea M., Helen L. Carlson, Catherine Lebel, Jacquie A. Hodge, Sean P. Dukelow, Jennifer A. Semrau, and Adam Kirton. 2017. "Sensory Tractography and Robot-Quantified Proprioception in Hemiparetic Children with Perinatal Stroke." Human Brain Mapping 38 (5): 2424-40. https://doi.org/10.1002/hbm.23530.

Kuo, Hsing Ching, Claudio L. Ferre, Jason B. Carmel, Jaimie L. Gowatsky, Arielle D. Stanford, Stefan B. Rowny, Sarah H. Lisanby, Andrew M. Gordon, and Kathleen M. Friel. 2017. "Using Diffusion Tensor Imaging to Identify Corticospinal Tract Projection Patterns in Children with Unilateral Spastic Cerebral Palsy.” Developmental Medicine and Child Neurology 59 (1): 65-71. https://doi.org/10.1111/dmen.13192.

Leemans, Alexander, Ben Jeurissen, Jan Sijbers, and Derek K. Jones. 2009. "ExploreDTI: A Graphical Toolbox for Processing, Analyzing, and Visualizing Diffusion MR Data." In 17th Annual Meeting of Intl Soc Mag Reson Med, 3537.

Leemans, Alexander, Jee Eun Lee, Mariana Lazar, and Aaron S. Field. 2007. "Diffusion Tensor Imaging of the Brain." Neurotherapeutics 4(3): 316-29. https://doi.org/10.1016/j.nurt.2007.05.011.

Lutkenhoff, Evan S., Matthew Rosenberg, Jeffrey Chiang, Kunyu Zhang, John D. Pickard, Adrian M. Owen, and Martin M. Monti. 2014. "Optimized Brain Extraction for Pathological Brains (OptiBET).” PLoS ONE 9 (12): 1-13. https://doi.org/10.1371/journal.pone.0115551.

Mai, J. K., and G. Paxinos. 2011. The Human Nervous System. Academic Press, New York.

Mailleux, Lisa, Inge Franki, Louise Emsell, Maarja-Liisa Peedima, Anna Fehrenbach, Hilde Feys, and Els Ortibus. 2020. "The Relationship between Neuroimaging and Motor Outcome in Children with Cerebral Palsy: A Systematic Review - Part B Diffusion Imaging and Tractography." Research in Developmental Disabilities 97. https://doi.org/10.1016/j.ridd.2019.103569.

Mailleux, Lisa, Katrijn Klingels, Simona Fiori, Cristina Simon-Martinez, Philippe Demaerel, Marlies 
Locus, Eva Fosseprez, et al. 2017. "How Does the Interaction of Presumed Timing, Location and Extent of the Underlying Brain Lesion Relate to Upper Limb Function in Children with Unilateral Cerebral Palsy?” European Journal of Paediatric Neurology 21 (5): 763-72. https://doi.org/10.1016/j.ejpn.2017.05.006.

Mori, Susumu, Kenichi Oishi, Hangyi Jiang, Li Jiang, Xin Li, Kazi Akhter, Kegang Hua, et al. 2008. "Stereotaxic White Matter Atlas Based on Diffusion Tensor Imaging in an ICBM Template." NeuroImage 40 (2): 570-82. https://doi.org/10.1016/j.neuroimage.2007.12.035.

Pagnozzi, Alex M., Nicholas Dowson, Simona Fiori, James Doecke, Andrew P. Bradley, Roslyn N. Boyd, and Stephen Rose. 2016. "Alterations in Regional Shape on Ipsilateral and Contralateral Cortex Contrast in Children with Unilateral Cerebral Palsy and Are Predictive of Multiple Outcomes." Human Brain Mapping 37 (10): 3588-3603. https://doi.org/10.1002/hbm.23262.

Pannek, Kerstin, Roslyn N. Boyd, Simona Fiori, Andrea Guzzetta, and Stephen E. Rose. 2014. "Assessment of the Structural Brain Network Reveals Altered Connectivity in Children with Unilateral Cerebral Palsy Due to Periventricular White Matter Lesions." NeuroImage: Clinical 5: 84-92. https://doi.org/10.1016/j.nicl.2014.05.018.

Perrone, Daniele, Jan Aelterman, Aleksandra Pižurica, Ben Jeurissen, Wilfried Philips, and Alexander Leemans. 2015. "The Effect of Gibbs Ringing Artifacts on Measures Derived from Diffusion MRI." NeuroImage 120: 441-55. https://doi.org/10.1016/j.neuroimage.2015.06.068.

Perruchoud, David, Micah M. Murray, Jeremie Lefebvre, and Silvio Ionta. 2014. "Focal Dystonia and the Sensory-Motor Integrative Loop for Enacting (SMILE)." Front Hum Neurosci 8: 458.

Porter, Robert, and Roger Lemon. 1993. Corticospinal Function and Voluntary Movement. Oxford: Clarendon Press.

Pruessmann, Klaas P., Markus Weiger, Markus B. Scheidegger, and Peter Boesiger. 1999. "SENSE: Sensitivity Encoding for Fast MRI.” Magnetic Resonance in Medicine 42 (5): 952-62. https://doi.org/10.1002/(SICI)1522-2594(199911)42:5<952::AID-MRM16>3.0.CO;2-S.

Randall, Melinda, Christine Imms, Leeanne M. Carey, and Julie F. Pallant. 2014. "Rasch Analysis of the Melbourne Assessment of Unilateral Upper Limb Function." Developmental Medicine and Child Neurology 56 (7): 665-72. https://doi.org/10.1111/dmen.12391.

Reid, Susan M., Elaine M. Meehan, Sarah J. Arnup, and Dinah S. Reddihough. 2018. "Intellectual Disability in Cerebral Palsy: A Population-Based Retrospective Study." Developmental Medicine and Child Neurology 60 (7): 687-94. https://doi.org/10.1111/dmcn.13773.

Rich, Tonya L., Jeremiah S. Menk, Kyle D. Rudser, Feyma Timothy, and Bernadette T. Gillick. 2017. "Less-Affected Hand Function in Children With Hemiparetic Unilateral Cerebral Palsy: A Comparison Study With Typically Developing Peers." Neurorehabilitation and Neural Repair 31 (10-11): 965-76. https://doi.org/10.1177/1545968317739997.

Roebuck-Spencer, Tresa M., Mattson Sarah N., Sarah Deboard Marion, Warren S. Brown, and Edward P. Riley. 2004. "Bimanual Coordination in Alcohol-Exposed Children: Role of the Corpus Callosum.” Journal of the International Neuropsychological Society 10 (4): 536-48. https://doi.org/10.1017/S1355617704104116.

Rose, Stephen, Andrea Guzzetta, Kerstin Pannek, and Roslyn Boyd. 2011. "MRI Structural Connectivity, Disruption of Primary Sensorimotor Pathways, and Hand Function in Cerebral Palsy." Brain Connectivity 1 (4): 309-16.

Scheck, Simon M., Jurgen Fripp, Lee Reid, Kerstin Pannek, Simona Fiori, Roslyn N. Boyd, and Stephen E. Rose. 2016. "Extent of Altered White Matter in Unilateral and Bilateral Periventricular White Matter Lesions in Children with Unilateral Cerebral Palsy." Research in Developmental Disabilities 55: 368-76. https://doi.org/10.1016/j.ridd.2016.04.007.

Scheck, Simon M., Kerstin Pannek, Simona Fiori, Roslyn N. Boyd, and Stephen E. Rose. 2014. "Quantitative Comparison of Cortical and Deep Grey Matter in Pathological Subtypes of Unilateral Cerebral Palsy." Developmental Medicine and Child Neurology 56 (10): 968-75. https://doi.org/10.1111/dmcn.12461.

Schober, Patrick, Christa Boer, and Lothar A. Schwarte. 2018. "Correlation Coefficients: Appropriate Use and Interpretation." Anesthesia \& Analgesia 126 (5): 1763-68. https://doi.org/10.1213/ANE.0000000000002864.

Simon-Martinez, Cristina, Ellen Jaspers, Lisa Mailleux, Els Ortibus, Katrijn Klingels, Nicole Wenderoth, and Hilde Feys. 2018. "Corticospinal Tract Wiring and Brain Lesion Characteristics 
in Unilateral Cerebral Palsy : Determinants of Upper Limb Motor and Sensory Function." Neural Plasticity 2018 (Article ID 2671613). https://doi.org/10.1155/2018/2671613.

Soares, José M., Paulo Marques, Victor Alves, and Nuno Sousa. 2013. "A Hitchhiker's Guide to Diffusion Tensor Imaging." Frontiers in Neuroscience 7 (7 MAR): 1-14. https://doi.org/10.3389/fnins.2013.00031.

Son, Su Min, Young Hwan Ahn, Joon Sakong, Han Ku Moon, Sang Ho Ahn, Ho Lee, In Kyu Yu, Youn Jeong Shin, and Sung Ho Jang. 2007. "Diffusion Tensor Imaging Demonstrates Focal Lesions of the Corticospinal Tract in Hemiparetic Patients with Cerebral Palsy." Neuroscience Letters 420 (1): 34-38. https://doi.org/10.1016/j.neulet.2007.04.054.

Staudt, Martin. 2010. "Brain Plasticity Following Early Life Brain Injury: Insights From Neuroimaging." Seminars in Perinatology 34 (1): 87-92. https://doi.org/10.1053/j.semperi.2009.10.009.

Staudt, Martin, Christian Gerloff, Wolfgang Grodd, Hans Holthausen, Gerhard Niemann, and Ingeborg Krägeloh-Mann. 2004. "Reorganization in Congenital Hemiparesis Acquired at Different Gestational Ages.” Annals of Neurology 56: 854-63. https://doi.org/10.1002/ana.10145.

Staudt, Martin, Wolfgang Grodd, Christian Gerloff, Michael Erb, Jutta Stitz, and Ingeborg KrägelohMann. 2002. "Two Types of Ipsilateral Reorganization in Congenital Hemiparesis: A TMS and FMRI Study." Brain : A Journal of Neurology 125: 2222-37. https://doi.org/10.1093/brain/awf227.

Tax, Chantal M.W., Willem M. Otte, Max A. Viergever, Rick M. Dijkhuizen, and Alexander Leemans. 2015. "REKINDLE: Robust Extraction of Kurtosis INDices with Linear Estimation." Magnetic Resonance in Medicine 73 (2): 794-808. https://doi.org/10.1002/mrm.25165.

Taylor, Neal, Patricia L. Sand, and Robert H. Jebsen. 1973. "Evaluation of Hand Function in Children." Archives of Physical Medicine and Rehabilitation 54: 129-35.

Thomas, Bejoy, Maria Eyssen, Ronald Peeters, Guy Molenaers, Paul Van Hecke, Paul De Cock, and Stefan Sunaert. 2005. "Quantitative Diffusion Tensor Imaging in Cerebral Palsy Due to Periventricular White Matter Injury," 2562-77. https://doi.org/10.1093/brain/awh600.

Tsao, Henry, Kerstin Pannek, Roslyn N. Boyd, and Stephen E. Rose. 2013. "Changes in the Integrity of Thalamocortical Connections Are Associated with Sensorimotor Deficits in Children with Congenital Hemiplegia." Brain Structure and Function 220 (1): 307-18. https://doi.org/10.1007/s00429-013-0656-x.

Tsao, Henry, Kerstin Pannek, Simona Fiori, Roslyn N. Boyd, and Stephen Rose. 2014. "Reduced Integrity of Sensorimotor Projections Traversing the Posterior Limb of the Internal Capsule in Children with Congenital Hemiparesis." Research in Developmental Disabilities 35 (2): 250-60. https://doi.org/10.1016/j.ridd.2013.11.001.

Verly, Marjolein, Robin Gerrits, Charlotte Sleurs, Lieven Lagae, Stefan Sunaert, Inge Zink, and Nathalie Rommel. 2018. "The Mis-Wired Language Network in Children with Developmental Language Disorder: Insights from DTI Tractography." Brain Imaging and Behavior, 1-12. https://doi.org/10.1007/s11682-018-9903-3.

Weinstein, Maya, Dido Green, Ronny Geva, Mitchell Schertz, Aviva Fattal-Valevski, Moran Artzi, Vicki Myers, et al. 2014. "Interhemispheric and Intrahemispheric Connectivity and Manual Skills in Children with Unilateral Cerebral Palsy." Brain Structure and Function 219 (3): 102540. https://doi.org/10.1007/s00429-013-0551-5.

Weinstein, Maya, Dido Green, Julian Rudisch, Ingar M. Zielinski, Marta Benthem-Muñiz, Marijtje L.A. Jongsma, Verity McClelland, et al. 2018. "Understanding the Relationship between Brain and Upper Limb Function in Children with Unilateral Motor Impairments: A Multimodal Approach." European Journal of Paediatric Neurology 22 (1): 143-54. https://doi.org/10.1016/j.ejpn.2017.09.012.

Zijdenbos, Alex P, Student Member, Benoit M Dawant, Richard A Margolin, and Andrew C Palmer. 1994. "Morphometric Analysis of White Matter Lesions in MR Images : Method and Validation" 13 (4). 